\title{
Size Effect in Photofield Emission
}

\author{
T. RADOŃ* \\ Institute of Experimental Physics, University of Wrocław, pl. Maxa Borna 9, 50-204 Wrocław, Poland
}

(Received November 19, 2009)

This paper is devoted to commemorate Prof. Dr. rer. nat. Christian Kleint (1926-2007)

whose pioneering work contributed greatly to photofield emission research.

\begin{abstract}
This paper contains a short reconsideration and explanation of the effect of photofield emission-current deviations. Theoretical considerations of the dynamic image-potential barrier did not yield a satisfactory result. A certain interpretation, suggested a few years ago, indicated that the photocurrent deviations reflect quantum-well states of the outer microcrystal of the apex of the field emitter. This point of view covers a variety of photocurrent deviations observed both for the integral field emission and for the one from a single crystal face as well as from a barium covered field emitter. It also includes the deviations observed for different photon energies and the deviations containing different apparent frequencies.
\end{abstract}

PACS numbers: 68.37.Vj, 73.20.At, 79.70.+q

\section{Introduction}

Combination of photoemission and field electron emission allows for measurement of the current of electrons excited to an energy below the vacuum level and hence to explore the electron states in the energy range between the Fermi and vacuum levels. An increasing interest in this method [1], so-called photostimulated field emission or photofield emission, caused the appearance of experimental results obtained by many authors [2-5]. Photofield emission characteristics reflect the density of states resulting from the band structure [6-9]. The photocurrent characteristics [9] and energy distributions of photofield electrons emitted from single crystal faces of the tungsten field emitter $[10,11]$ show a very good agreement with the exactly calculated band structure [12].

Some measurements of photocurrent characteristics have revealed a new unexpected effect. The integral photofield emission characteristics [2, 13], particularly the ones measured from a single crystal face [14], show distinct regular photocurrent deviations (oscillations) in a wide range of electric field applied. There have been made efforts to explain this effect taking into account the dynamic image potential instead of the classical image potential [15-17], but those attempts did not yield a satisfactory explanation.

In this paper, the observed photocurrent deviations are considered taking account of the dimension of the microcrystal which represents the top of the field emitter.

\footnotetext{
* corresponding author; e-mail: radon@ifd.uni.wroc.pl
}

An attempt to approach the explanation was made for a tungsten field emitter [18] and confirmed by the results obtained for a tantalum field emitter [19]. All the results containing such photocurrent oscillations are reviewed taking into consideration the new interpretation.

\section{The microcrystal of the field emitter tip}

Apex diameter of the chemically or electrochemically etched part of the field emitter is very small and can be compared with the dimension of underlying microcrystals of the tip. The etched part of the field emitter is so narrow that may be treated as microcrystals connected in a pile, which seems to be confirmed in studies by scanning electron microscopy [20]. Then the top of an emitter usually contains one microcrystal (Fig. 1a) and the field emission pattern shows the symmetry of a single crystal. This microcrystal can be more or less etched up during preparation of the field emitter and its size can be different. Such a very small microcrystal may not cover the top of the microcrystal lying below (Fig. 1b) then the field emission pattern shows two different microobjects. Mostly, after heating the emitter at high temperature, the observed pattern shows the symmetry of a single crystal. This means that the apex microcrystal has grown at the cost of the microcrystal lying below or the microcrystal at the top has received structure of the adjacent microcrystal.

\section{The photocurrent deviations}

According to the proposed concept [18], the source of the photocurrent deviations is the microcrystal situated 


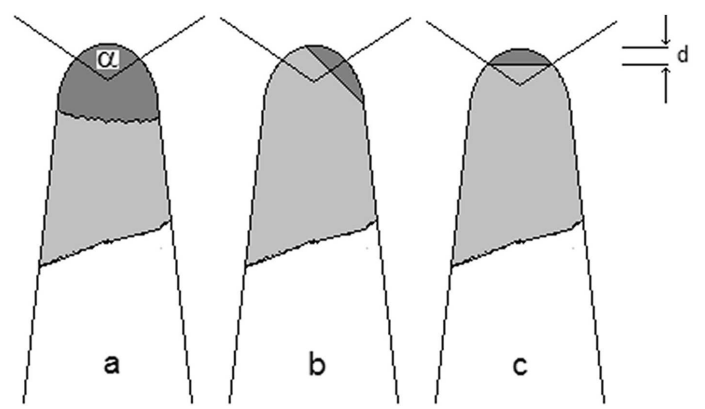

Fig. 1. Microcrystals of the field emitter: (a) an arbitrary sketch of two microcrystals in a field emitter, $\alpha$ is an angle of electron emission; (b) two microcrystals can create a field emission pattern representing parts of two microcrystals; (c) the thin emitting microcrystal, $d$ is the smallest thickness of the microcrystal which gives the complete symmetry of a field emission pattern.

at the top of the field emitter tip. An ideal form of the thin microcrystal is shown in Fig. 1c. This microcrystal may be treated as a quantum well for electrons, the adjacent microcrystal is separated by the grain boundary which is not entirely transparent for electrons. The electron states in the quantum well are regularly ordered $[21,22]$ and occupied via photoexcitation like the other empty states in the band structure between the Fermi and vacuum levels. Then the photocurrent contains the electrons which reflect the density of states concerning the band structure and other electrons regularly distributed in energy within the quantum well. The two groups of electrons are measured together and the small shoulders present in the photocurrent characteristic are the oscillations indicating the quantum well states.

A good example of such photocurrent deviations is given by a curve of Fig. 2 [18], elaborated on the basis of an early measurement [23]. Originally, those points in the curve were treated as the statistical dispersion expected for the measurement of very small photocurrents. Because the measurements were carried out under very good vacuum conditions, those points must have revealed also regular deviations from the smooth curve. After subtracting the thermocurrent from the measured photocurrent [24] a true photocurrent curve could be obtained, which also shows the deviations, and after differentiation the regularly spaced maxima are obtained; this energy distribution of electrons is shown in inset in Fig. 2.

\section{Discussion}

This explanation of the maxima indicating the energy peaks of electron states is closely connected with the results obtained for a thin titanium microcrystal grown on the tungsten field emitter. It is experimentally shown that a twice thinner microcrystal of titanium reveals twice less maxima of the density of states [25]. The titanium microcrystal at the apex of the tungsten field emitter is separated by the grain boundary, which forms

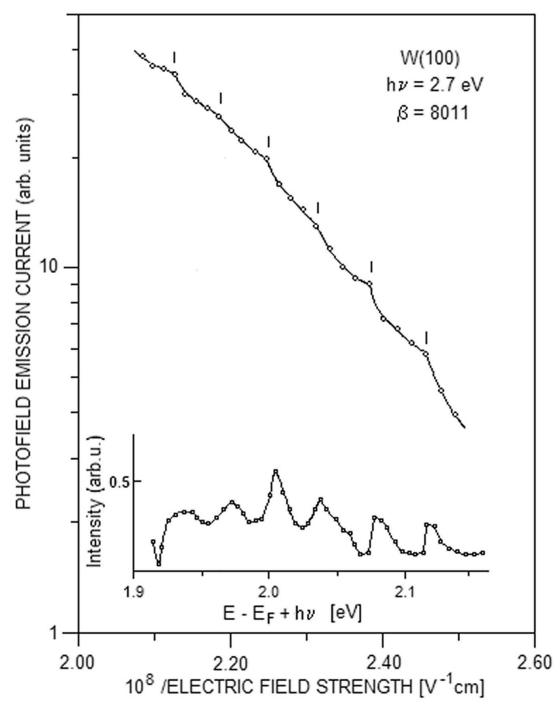

Fig. 2. A photofield emission characteristic of the (100) region of tungsten [18]. Photocurrent was measured when the electric vector of $p$-polarized light was nearly perpendicular to the emitting surface, the angle of incidence of the light beam onto the plane was about $39^{\circ}$. Inset shows the energy distribution of electrons after subtracting the thermocurrent from the measured photocurrent [24]. The irradiance at the tip was less than $100 \mathrm{~W} / \mathrm{cm}^{2}$.

a particular trap for electrons. Therefore the titanium microcrystal can be treated as a quantum well for electrons.

Likewise, the microcrystal forming the apex part of the homogenous field emitter is also separated from its "substrate" by a "grain boundary", which may be treated as the quantum well for electrons. When the microcrystal is suitably thin (i.e. when $d$ is sufficiently small, see Fig. 1c), it is possible to observe the regularly distributed shoulders in the photocurrent curve (Fig. 2) and hence the regularly spaced maxima (peaks) in the energy distribution of electrons. The various results obtained either from both the clean and the adsorbate covered tip surface or from a single face of the field emitter were obtained by using different photoexcitation energies. It seems that all those can now easy be explained in terms of the thickness of the microcrystal emitting the electrons.

In the photocurrent curve reported by Lee and Reifenberger [14], the regularly spaced maxima change their spacing in the way characteristic to quantum well states. This is clearly observed because the photocurrent curve for a single crystal face is obtained in a wide energy range of $0.9 \mathrm{eV}$. The maxima are closer spaced $(\approx 0.04 \mathrm{eV})$ for higher energy levels and considerably more spaced $(\approx 0.08 \mathrm{eV})$ for lower ones. The expected tendency is also noticeable in the photocurrent curve obtained from the barium covered tip of tantalum [26], but the spacing $(\approx 0.004 \mathrm{eV})$ is about 10 times smaller (part of the longer photocurrent curve is presented in Fig. 3). The greater spacing of maxima $(0.04-0.08 \mathrm{eV})$ in the former 
case indicates a smaller thickness of the electron-emitting microcrystal, and the smaller spacing in the latter case indicates such a thicker microcrystal. In accordance with the proposed interpretation the barium adsorbate caused only lowering the work function.

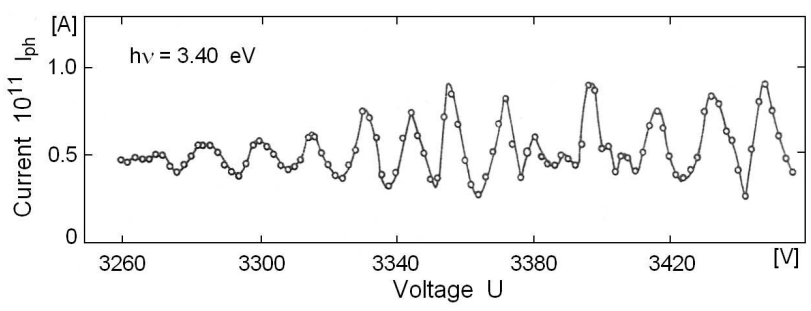

Fig. 3. Part of three total photocurrent oscillations under axial irradiation of the tungsten field emitter by a mercury superpressure lamp [13]. The spectral composition was given by monochromatic filters. The irradiation of the tip was of the order $1 \mathrm{~W} / \mathrm{cm}^{2}$.

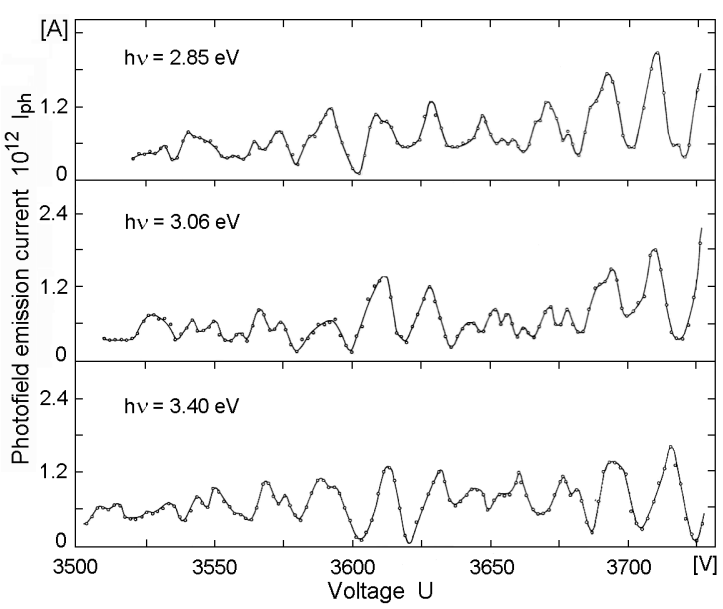

Fig. 4. Part of total photofield emission current oscillations of the barium covered tantalum field emitter [26] (work function $\varphi=3.31 \mathrm{eV}$ ) under axial illumination by mercury lamp using monochromatic filter for $h \nu=3.40 \mathrm{eV}$. The irradiation density was of the order $1 \mathrm{~W} / \mathrm{cm}^{2}$. The emitter was cooled by liquid nitrogen.

Also, different photon excitation energies do not change the character of the measured curves, see Fig. 4 [13]. The three curves are well reproduced, although their quantum energies are markedly different. There are visible a few great maxima obtained at higher voltages, then some smaller maxima, greater maxima and again small maxima appear at the lowest voltages (this character of the curves suggests that the maxima are originated from two or more emission sites; this is imaginable because the photocurrents are measured from the whole field emitting surface). If the maxima's spacing is really connected with the thickness of the emitting microcrystal, then the measurement results both from the whole surface and from a single face of the field emitter tip should be nearly the same. It is difficult to estimate the thickness of the microcrystal creating the top of the field emitter and there is not, so far, any direct evidence reported for the new interpretation.

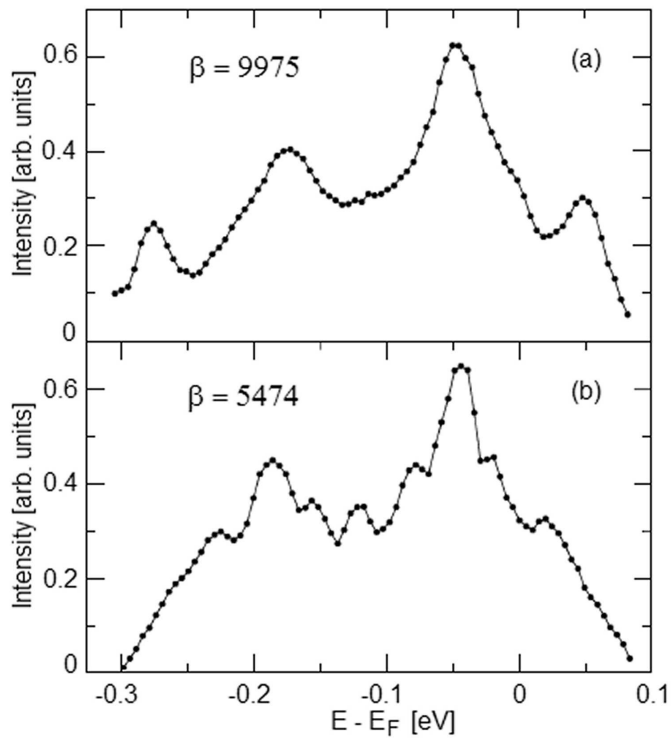

Fig. 5. Energy distributions of photoelectrons emitted from the tantalum (111) face characterized by two different values of the geometrical factors: (a) $\beta=9975 \mathrm{~cm}^{-1}$, (b) $\beta=5474 \mathrm{~cm}^{-1}$. The photocurrent curves were obtained using photon energy $h \nu=1.96 \mathrm{eV}$ and $p$ -polarized light [19].

Until 1984, measurements revealing the photocurrent deviations were reported for a few (about five) different field emitters, four of which were tungsten tips and one was a barium covered tantalum tip. In three cases $[2,13,25]$ the photocurrent deviations were noticed accidentally, and it is an additional argument indicating the microcrystal at the top of field emitter to be the source of the photocurrent oscillations. They were observed during the conventional measurement of the photocurrent curve, when the emitting microcrystal was suitably thin. In one of our early measurements, photocurrent oscillations were not observed after heating the tungsten field emitter at a high temperature, although the oscillations were measured before heating. Probably a neighboring microcrystal increased its dimension at the cost of the microcrystal at the top of the field emitter tip, or on the contrary, the apex microcrystal increased. Then the thick microcrystal might have produced the closely spaced maxima, but those were practically difficult to observe. The grain boundary between the apex microcrystal and its substrate microcrystal may move or disappear during heating of the field emitter. By this means it is possible to change the thickness of the apex microcrystal and to observe the change of the maxima spacing. This anticipation has been proved and confirmed for the tantalum field emitter [19]. For the small tip radius $r$, otherwise for the great geometrical coefficient $\beta$ 
$(\beta \approx 1 / r)$, we observe four maxima $\left(\beta=9975 \mathrm{~cm}^{-1}\right)$ and for the smaller $\beta$, after heating the tip at high temperature, we observe eight maxima $\left(\beta=5474 \mathrm{~cm}^{-1}\right)$, see Fig. 5. This means, that the grain boundary between the two microcrystals is displaced from the top of the tip, and the thickness of the emitting microcrystal is greater now. Moreover, it has appeared that polarization of the light beam does not change the apparent frequency of the photocurrent oscillations, and the general shapes of two curves reflect different band structures of bulk metal and that of the metal surface, but the small maxima remain practically unchanged [19].

Small sharp peaks in the field emission energy distribution from silicon have also been observed [27]. Authors suggested, that it might be a result of an emission from the quantized states of a potential well.

Theoretical prediction that photofield emission oscillations originate from a limited surface area of the emitter tip was given by Modinos and Kleint [28], however, any measurements from such small surface areas have not been reported so far.

\section{Acknowledgments}

The author wishes to thank S.A. Surma for helpful discussions, and P. Hądzel for technical assistance.

\section{References}

[1] H. Neumann, C. Kleint, Ann. Phys. 27, 237 (1971).

[2] J. Wysocki, C. Kleint, Phys. Stat. Sol. A 20, K57 (1973).

[3] M.J.G. Lee, Phys. Rev. Lett. 30, 1193 (1973).

[4] Y. Teisseyre, R. Coelho, R. Haug, Surf. Sci. 52, 120 (1974).

[5] R. Liu, G. Ehrlich, R.S. Polizzotti, J. Vacuum Sci. Technol. 11, 276 (1974).

[6] J. Wysocki, C. Kleint, Acta Phys. Pol. A 48, 157 (1975).
[7] Y. Teisseyre, R. Haug, R. Coelho, Surf. Sci. 75, 592 (1978).

[8] T. Radoń, C. Kleint, Surf. Sci. 60, 540 (1976).

[9] T. Radoń, Surf. Sci. 100, 353 (1980).

[10] D. Venus, M.J.G. Lee, Phys. Rev. B 34, 4449 (1986).

[11] D. Venus, M.J.G. Lee, Surf. Sci. 171, 477 (1986).

[12] N.E. Christensen, B. Feuerbacher, Phys. Rev. B 10, 2349 (1974).

[13] T. Radoń, C. Kleint, Ann. Phys. 7, 239 (1977).

[14] M.J.G. Lee, R. Reifenberger, Surf. Sci. 70, 114 (1978).

[15] R. Reifenberger, D.L. Haavig, C.M. Egert, Surf. Sci. 109, 276 (1981).

[16] C. Schwartz, M.W. Cole, Surf. Sci. 115, 290 (1982).

[17] C.M. Egert, R. Reifenberger, Surf. Sci. 145, 159 (1984).

[18] T. Radoń, L. Jurczyszyn, P. Hądzel, Surf. Sci. 513, 549 (2002).

[19] P. Hądzel, L. Jurczyszyn, T. Radoń, Appl. Surf. Sci. 222, 243 (2004).

[20] M. Drechsler, A. Piquet, R. Uzan, Vu Thien Binh, Surf. Sci. 14, 457 (1969).

[21] V.N. Lutskii, Phys. Stat. Sol. A 1, 199 (1970).

[22] M. Jałochowski, Prog. Surf. Sci. 48, 287 (1995).

[23] T. Radoń, S. Jaskółka, Surf. Sci. 231, 160 (1990).

[24] T. Radoń, P. Hądzel, M.J.G. Lee, Z.A. Ibrahim, I.C.L. Chow, Surf. Sci. 549, 103 (2004).

[25] P. Hądzel, T. Radoń, M.J.G. Lee, J.C.L. Chow, Surf. Sci. 442, 36 (1999).

[26] C. Kleint, T. Radoń, Acta Universitatis Wratislaviensis 471, 30 (1979).

[27] M.H. Herman, T.T. Tsong, Phys. Rev. Lett. 48, 1029 (1982).

[28] A. Modinos, Ch. Kleint, Solid State Commun. 50, 651 (1984). 